Workshop in Frankfurt

Studien richtig

planen und aufsetzen

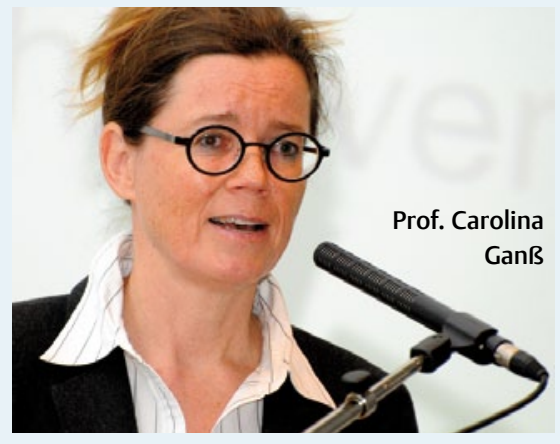

Unterstützt von der neuen Fortbildungsinitiative „GABA Oral Health Network“ lädt die ebenfalls neu ins Leben gerufene DGPZM (Deutsche Gesellschaft für Präventivzahnmedizin) zu einer Weiterbildung nach Frankfurt am Main ein. „Clinical Studies: From the idea to first patient's first visit" lautet der Titel des englischsprachigen Workshops mit hochkarätigen Referenten am Freitag, 13. Juli 2012, im Museum für Kommunikation. Die Teilnehmer werden dort Informationen rund um die Planung, das Aufsetzen und die Veröffentlichung wissenschaftlicher Studien erhalten. Der Inhalt spannt einen Bogen von den rechtlichen Rahmenbedingungen über praktische Aspekte bis hin zur korrekten Zusammenfassung wissenschaftlicher Studien. Die Leitung der Veranstaltung obliegt der Präsidentin der DGPZM, Prof. Carolina Ganß. Die weiteren Referenten sind: Dr. Gabriele Pohlig, Tropeninstitut, Basel, Prof. Ian Needleman, UCL; Eastman Dental Institute, London, PD Dr. Christian Heumann, LMU, Abteilung für Statistik, München.

Der Workshop richtet sich an wissenschaftliche Mitarbeiter und Forscher von Universitäten. Die Teilnehmerzahl ist auf maximal 40 begrenzt. Beginn der Vorträge ist um 13 Uhr, Ende um 19 Uhr. Ab 20 Uhr klingt die Veranstaltung mit einem Abendessen aus. Die Kursgebühr beträgt 180,-€ inkl. Material, Verpflegung während der Veranstaltung, Nachtessen und Übernachtung (ermäßigte Gebühr von 100,-€ für Doktoranden auf Anfrage möglich). Anmeldung bitte bis spätestens 5. Juni 2012 bei: heike.wendrich@dentist. med.uni-giessen.de.

Nach einer Pressemitteilung der

GABA GmbH, Lörrach

E-Mail: info@gaba.gmbh

Preisverleihung

\title{
Praktikerpreis an Dr. Christian Krupp
}

Erneut hat die Deutsche Gesellschaft für Zahnerhaltung (DGZ) auf ihrer Jahrestagung den GABA Praktikerpreis der DGZ für herausragende praxisorientierte $\mathrm{Ar}$ beiten auf dem Gebiet der Zahnerhaltung verliehen. Die Auszeichnung ging diesmal an Dr. Christian Krupp (Zahnarztpraxis Dr. Bargholz \& Partner, Hamburg) für seinen Vortrag „Orthograde Entfernung retrograder Wurzelkanalfüllungen - eine Technikbeschreibung“, präsentiert auf der Jahrestagung der DGZ im vergangenen Jahr. Der Preis ist mit 3000,-€ dotiert.

Einstimmig hatte sich die Jury - bestehend aus Prof. Gottfried Schmalz, Universität Regensburg, Dr. Sabine Friese, Kirchhain, und Dr. Florian Eichelsbacher, Frankfurt/Main - für Dr. Krupps Vortrag entschieden. Insgesamt waren 9 Vorträge angemeldet.

An 4 exemplarischen Falldarstellungen illustrierte und erläuterte der Preisträger in seinem Vortrag das technische Vorgehen der vollständigen Entfernung retrograder Füllmaterialien. Anhand der Darstellung eines Falles, bei dem nur eine unvollständige Entfernung des Füllmaterials möglich war, beleuchtete und bewertete er kritisch die therapeutischen Grenzen des Vorgehens. Zudem wies er darauf hin, dass aufgrund der Daten aus der vorliegenden Literatur zum gegenwärtigen Zeitpunkt die rein orthograde

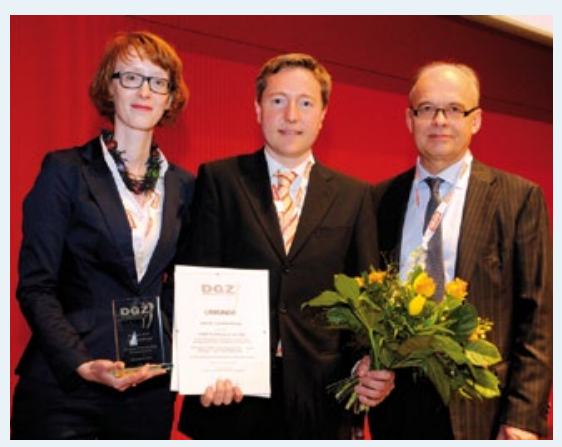

V.I.n. r.: Dr. C. Brose, Dr. C. Krupp, Prof. W. Raab.

Revision nicht erfolgreicher Wurzelspitzenresektionen noch nicht als Standardtherapie empfohlen werden könne. Bislang lägen zu dieser Thematik nur wenige Einzelfalldarstellungen und keine Langzeitstudien vor.

Der Praktikerpreis wird jährlich von der DGZ gemeinsam mit GABA, Spezialist für orale Prävention, vergeben. Wichtige Kriterien für die Vergabe sind die Qualität der Dokumentation, die Komplexität der Problemdarstellung, der Vortragsstil und die Praxisrelevanz. „Mit dem Praktikerpreis wollen wir die klinische Tätigkeit auf dem gesamten Gebiet der Zahnerhaltung unterstützen und den Austausch zwischen Praxis und Wissenschaft fördern“, erläutert Dr. Robert Eichler, Leiter Medizinische Wissenschaften bei GABA.

Dieser Beitrag ist entstanden mit freundlicher Unterstützung der GABA GmbH, Lörrach.

\section{Zahnfleischprobleme}

\section{Testwochen im Mai gestartet}

Für viele Patienten mit Gingivitis und Parodontitis sind Produkte der Marke meridol die Empfehlung schlechthin. Da ihre Wirkung nicht nur in zahlreichen klinischen Studien bewiesen, sondern für die Verwender auch eindeutig spürbar ist, ist beispielsweise die meridol Zahnpasta bei Zahnfleischproblemen die Nummer 1 in der Zahnarztempfehlung. Eine deutliche Sprache spricht das Ergebnis eines Patiententests in der Schweiz im Jahr 2011: 93\% der insgesamt 77 Teilnehmer bestätigten, nach spätestens 21 Tagen frei von Zahnfleischproblemen gewesen zu sein. Hersteller GABA ruft nun auch in Deutschland zu einer großen Testaktion auf: Von
Mai bis August bieten die „meridol Testwochen“ Verwendern die Möglichkeit, die Produkte der Marke zu testen. Dabei sollen sich möglichst viele Patienten mit Zahnfleischproblemen beteiligen. Anschließend haben die Tester die Möglichkeit, auf www. meridol.de ihre Erfahrungen mitzuteilen und die Produkte zu bewerten. Mit etwas Glück gewinnen sie eine Traumreise oder einen von 100 Wellness-Preisen. Alle Zahnarztpraxen in Deutschland sind aufgerufen, sich an der Aktion zu beteiligen und ihre Patienten zum Mitmachen zu animieren.

Nach einer Pressemitteilung der

GABA GmbH, Lörrach

E-Mail: info@gaba.com 